\title{
INFORMATIVE COLLISION WARNINGS: EFFECT OF MODALITY AND DRIVER AGE
}

\author{
Mujthaba Ahtamad ${ }^{1}$, Robert Gray ${ }^{1}$, Cristy $\mathrm{Ho}^{2}$, Nick Reed ${ }^{3}$ \& Charles Spence ${ }^{2}$ \\ ${ }^{1}$ University of Birmingham, ${ }^{2}$ University of Oxford, ${ }^{3}$ Transport Research Laboratory \\ United Kingdom \\ E-mail:m.ahtamad@bham.ac.uk
}

\begin{abstract}
Summary: Research has revealed that when drivers are presented with an informative tactile collision warning, they are able to produce faster braking reaction times (BRTs) which may potentially reduce the likelihood and severity of rear-end collisions. To expand on this research, we investigated the effectiveness of unimodal (tactile) and multisensory (audiotactile) informative collision warnings for younger and older drivers. In line with our previous results, driver BRTs were significantly faster when they were presented with an informative signal as compared to a non-informative signal and a control condition in which no warnings were presented. The results also revealed that the unimodal informative warning was just as effective as the multisensory warning. Intriguingly, older drivers exhibited faster BRTs than younger drivers, and were significantly faster following the presentation of multisensory warning signals. Finally, this study identifies the need to compare new configurations of informative tactile collision warning signals.
\end{abstract}

\section{INTRODUCTION}

Road Traffic Collisions (RTCs) are a preventable burden on society (Murray et al., 2012). One of the most common types of RTC is the rear-end collision, accounting for nearly a third of all vehicle related accidents (Lao et al, 2014) and 43\% of injuries (Watanabe et al., 2000). In order to reduce their likelihood and severity, collision warning systems are increasingly being developed as part of Advanced Driver Assistance Systems (ADAS) that are becoming available in new vehicles. For instance, Forward Warning Collision Systems (FWCS) are designed to redirect the attention of the driver towards any potential on-coming collisions (Maltz \& Shinar, 2007). Research into FWCS has focussed on identifying a warning signal which can effectively facilitate rapid braking reaction times (BRTs) from drivers. This has led researchers to explore which of the human senses may be best suited for perceiving collision warning signals in order to try to ensure that the most appropriate behavioural response is produced.

In recent years, a range of sensory modalities have been explored. For example, visual warnings such as icons on the vehicle dashboard (Lee et al., 2002; Baldwin \& Lewis, 2014), auditory signals in the form of tones (Gray, 2011), including speech, car horn, and screeching tyre sounds (Graham, 1999; McGehee et al., 2002; Ho \& Spence, 2005, 2008; Ho, Santangelo, \& Spence, 2009) have previously been researched. The efficacy of auditory interventions including manipulations of the playback from the radio (e.g., panning audio between left and right speakers), and preventing any additional stimuli from being introduced into the cabin and potentially causing further distraction have also been investigated (Fagerlönn, Lindberg, \& Sirkka, 2012). Tactile warning signals have also been developed that use vibrational warning cues to alert or redirect distracted drivers (Van Erp, 2005; Ho, Spence \& Gray, 2013; Chun et al., 2014). Due to there already being a range of sounds in the vehicle cabin, any benefits from an 
auditory FWCS may possibly be reduced and auditory signals may be distorted. The tactile modality shows promise in terms of being less influenced by sound distractions such as talking on a telephone (Mohebbi, Gray \& Tan, 2009). As a result, the current focus of our study is aimed at tactile and multisensory (specifically audiotactile) collision warning signals.

Informative Collision Warnings. A relatively recent and promising innovation is the development of informative FWCS. Informative warnings signals are designed to alert the driver and also carry additional information about the event outside of the vehicle. For example, the signals, depending on the modality used to present them, can be configured within certain parameters to relay a sense of urgency to the driver. A visual warning may make use of different colours and font sizes to increase the perceived urgency of the signal (Adams \& Edworthy, 1995) or it may even be designed to flash or to move in order to capture the driver's attention (Laughery, 2006). Auditory warnings can be adjusted to vary the perceived urgency by creating a looming signal for which the intensity increases with the approach of an on-coming vehicle (Gray, 2011). These looming warnings were found to produce faster BRTs than comparable non-informative auditory signals and produced fewer unnecessary braking responses under false alarm conditions than a car horn warning signal.

Similarly, vibrotactile warnings can also be configured to relay the sensation of an object approaching the driver. So, for example, Ho, Gray, and Spence (2014) created a vibrotactile warning signal consisting of the sequential activation of three tactors aligned vertically on the abdomen. When the rate of apparent motion was linked to the closing velocity and the direction of apparent motion was towards the head, this warning resulted in significantly shorter BRTs (see also Gray, Ho \& Spence, 2014). Similarly, Meng et al. (2015a) presented drivers with a FWCS which consisted of vibrotactile signals presented sequentially first at the hands and then immediately to the torso. These dynamic or informative warnings revealed a significant advantage in reducing driver reaction times in contrast to signals that were activated simultaneously or signals that simulated motion away from the driver (see also Meng et al., 2015b).

\section{Aims of the present study}

The goal of the present study was to expand on our previous research on informative auditory and tactile warning signals with two particular aims. First, since all of our previous studies have involved younger drivers, we sought to investigate the effect that driver age has on the effectiveness of informative collision warnings. Previous research investigating the effect of driver age on the effectiveness of collision warnings have differential benefits for older and younger drivers, with older drivers in some cases actually benefiting more from collision warnings than younger drivers (Kramer et al., 2007; Porter et al., 2008; Chun et al., 2012). Therefore, it is interesting to ask whether there will be similar age effects for informative collision warning signals. The second aim of the present study was to investigate whether multisensory informative warnings produce any additional BRT benefits relative to unimodal warnings, as have been shown in the case of non-informative warnings (e.g., Ho, Reed \& Spence, 2007). 


\section{METHODS}

\section{Participants}

32 participants (9 female, 23 male, Mean Age of 46, SD = 20) took part in the study. The participants were sub-divided into two equal groups, younger drivers (Range $=18-35$ years, Mean $=27, \mathrm{SD}=5)$ and older drivers $($ Range $=60-75$ years, Mean $=66, \mathrm{SD}=5)$. All drivers had a valid UK driving license.

\section{Apparatus and Materials}

A high fidelity driving simulator based on a Honda Civic hatchback car was used. The driving environment was projected at a resolution of up to $1920 \times 1457$ pixels onto each of three forward screens giving a $210^{\circ}$ horizontal forward field of view and a rear screen providing a $60^{\circ}$ rear field of view, thus permitting the normal use of the rear view and two side mirrors. The driving simulation was generated by the SCANeR Studio software (OKTAL, France). A rural, bidirectional, single carriageway road was used as the environment setting with a speed limit of $60 \mathrm{mph}$ throughout. Drivers were required to accelerate and to follow a lead vehicle, maintaining a 2-second gap behind the lead vehicle and the driven vehicle. The lead vehicle's brake lights were deactivated. This was a similar scenario to those used in previous studies (e.g., Ho, Reed \& Spence, 2007).

Warning Signals. All of the vibrotactile stimuli used in this study were presented to the participants via tactors (VBW32, Audiological Engineering Corp., Somerville, MA, USA) that were fastened as pairs to: 1) hands (one tactor on the back of each hand) and 2) the waist (one tactor on each side of the participant's abdomen). The tactors were either activated sequentially (informative) for $150 \mathrm{~ms}$ at the hands followed by an immediate activation at the waist for 150 $\mathrm{ms}$, or were simultaneously activated for $300 \mathrm{~ms}$ (non-informative). The following signals were investigated and were used as conditions in the study.

- Vibrotactile non-informative - All tactors were simultaneously activated.

- Vibrotactile informative - Tactors to the hands were activated first and the waist second.

- Multisensory informative - A looming auditory warning (Gray, 2011) was presented together with a simultaneous informative vibrotactile signal.

- No warning (Control) - No signals were presented.

\section{Design and Procedure}

A mixed-methods design was used. The within-participants factor was the type of warning. Each of the 4 conditions was tested in separate blocks of 21 trials. These were comprised of 18 trials in which the warning (if present) was reliable (i.e., warning activated at TTC $=3 \mathrm{~s}$ ) and 3 trials in which the warning was unreliable (i.e., warning activated at $\mathrm{TTC}=7 \mathrm{~s}$ ). The order of reliable and unreliable trials was randomised. The between-participants factor was age group (younger vs. older). The experiment lasted for approximately $90 \mathrm{~min}$. The participants were requested to keep both hands on the steering wheel. During each trial, the drivers were instructed to accelerate in order to catch up with the lead vehicle and then to maintain a $2 \mathrm{~s}$ time headway (TH). The lead 
vehicle travelled at a speed ranging between 35 and $45 \mathrm{mph}$ with speed changes once every $5 \mathrm{~s}$, on average. At a random time between $20-40 \mathrm{~s}$ after the beginning of a trial, the lead vehicle braked suddenly with a $-6 \mathrm{~m} / \mathrm{s}^{2}$ deceleration rate and came to a complete stop. These speed changes were designed to reduce the tendency for drivers to anticipate the stopping event. The drivers were instructed to brake in order to avoid a collision with the lead vehicle and not to leave the lane (any trials for which this occurred were discarded). Each trial ended when the participant's car came to a complete standstill and/or collided with the lead vehicle. During each block the type of warning signal remained constant.

Data Analysis. The dependent variable of interest was the BRT, defined as the time after the onset of a critical braking event when the participant initiated a braking response by depressing the brake pedal to over $1 / 3$ of its complete depression. BRTs were analyzed using a mixed analysis of variance (ANOVA) with the warning type (no warning, vibrotactile non-informative, vibrotactile informative, and multisensory informative) and age group (younger and older) as the experimental factors.

\section{RESULTS}

Effect of Warning Type: Mean BRTs for the different warning conditions and groups are presented in Figure 1.

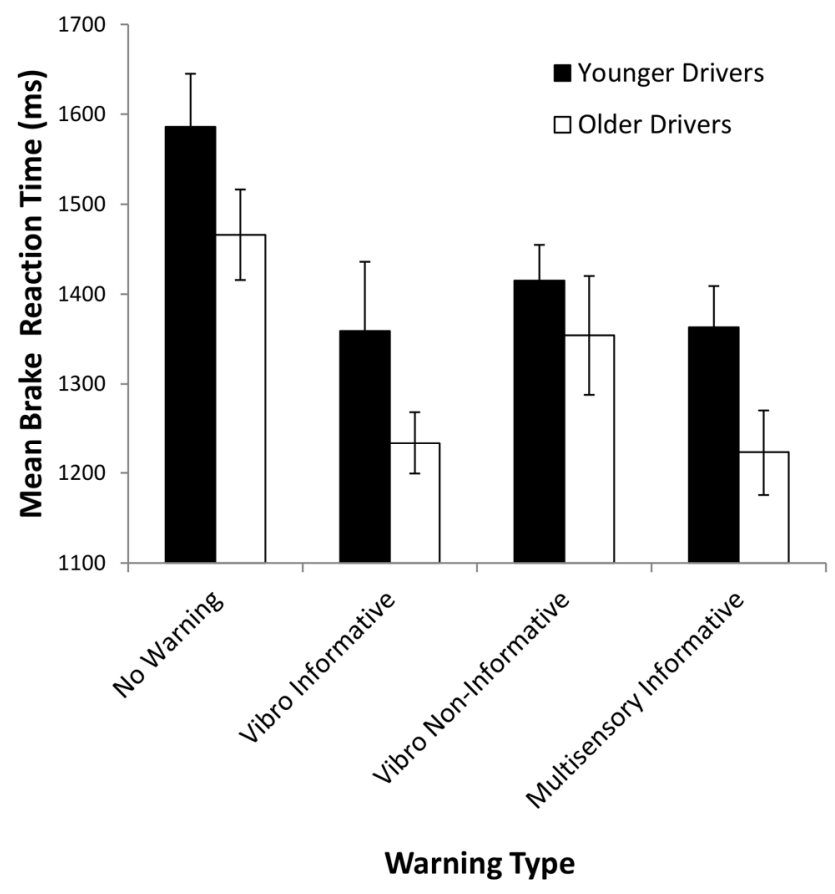

Figure 1 - Mean BRTs. Error bars are standard errors

The ANOVA revealed a significant main effect of warning signal, $F(3,87)=24.30, p<.001, \eta_{\mathrm{p}}^{2}=$ .75. Post-hoc comparisons with an applied Bonferroni correction ( $p$ adjusted $=.008$ ) revealed that participants braked significantly more rapidly following the presentation of any warning signal compared to no warning signal, all $p$ values were significant $(<.001)$. Further comparisons revealed that relative to the non-informative vibrotactile warnings, the multisensory informative 
warning resulted in significantly faster BRTs, $\mathrm{t}(31)=3.1, p=.004$ and informative vibrotactile warnings resulted in a reduction in BRT that was marginally significant, $\mathrm{t}(32)=2.4, p=0.02$. There was no significant difference in BRTs between the vibrotactile informative and multisensory informative warning signals $(p>.5)$.

Effect of Driver Age: The ANOVA further revealed a significant main effect of age group, $F(1$, $29=4.95, p=0.034, \eta_{p}^{2}=.15$. As can be seen in Figure 1, this occurred because the older driver group in our study generally produced shorter BRTs relative to the younger driver group. Posthoc comparisons with Bonferroni correction ( $p$ adjusted $=0.012$ ) revealed that older drivers had significantly shorter BRTs than younger drivers in the multisensory condition, $\mathrm{t}(30)=2.62$, $p=0.011$. There was no significant difference between the age groups for any of the other warning conditions $(p$ all $>0.1)$. The age $\times$ warning type interaction in the ANOVA was not significant $(p>0.5)$.

\section{DISCUSSION}

Our recent research demonstrates that informative warning signals show promise as a means of effectively reducing driver RTs in responding to a potential collision. The goals of the present study were to further advance this by investigating relative effect of unimodal and multisensory informative collision warnings and driver age on the effectiveness of informative warnings. In previous research, a non-informative multisensory (audiotactile) warning was found to be the most effective in terms of producing significantly faster BRTs in contrast to non-informative unimodal warnings (Ho, Reed \& Spence, 2007). Interestingly, the results of the present study, which used the same simulator architecture, revealed that the informative unimodal warning was just as effective as an informative multisensory warning in reducing driver BRTs significantly.

It should be noted that the present experiment was run on a higher fidelity simulator than used in the majority of our previous experiments, particularly those evaluating informative vibrotactile warnings (Meng, et al., $2015 \mathrm{a}, \mathrm{b}$ ). Given that there can be limitations when drawing inferences about real driving from laboratory experiments (Ho, Gray \& Spence, 2014b), an important step in validating the effectiveness of informative warnings is ensuring that they produced similar results when the Noise, Vibration and Harshness (NVH) characteristics introduced into the vehicle via the cabin, through the pedals, seat, and steering wheel are more similar to real driving.

Another limitation that was dealt with in the present study was to address the lack of older drivers taking part in much of the earlier research in this area. Previous research has found mixed results concerning the effectiveness of warning signals for experienced and older drivers. In one example, delivering vibrational signals on a seatbelt and steering wheel was generally found to be more effective for younger drivers, producing faster RTs than older drivers (Chun et al., 2012). Contrary to our expectations, we identified an age effect whereby the older drivers in our study responded more rapidly than the younger drivers in all of the conditions. Interestingly, Kramer et al. (2007) also found that older drivers could benefit just as much, if not more than their younger counterparts when responding to collision warnings. However, further research will be required in order to understand how the different age groups perceived each of the warnings and indeed whether or not multisensory warning signals place greater demands on 
drivers in comparison to the unimodal signal. As both informative warning conditions (multisensory/unimodal) gave rise to similar BRTs, it will be valuable to understand which of the signals were perceived subjectively to be the most urgent. Finally, research is still needed to explore alternate locations on the body and/or activation sequences of informative tactile signals that can be effective in reducing driver BRTs.

\section{CONCLUSIONS}

These results from the advanced vehicle simulator validate previous laboratory findings and also reveal that an informative unimodal collision warning is just as effective as a multisensory collision warning in facilitating driver BRTs. Furthermore, the informative signals initiated faster RTs from older drivers than for younger drivers. The study shows further benefits for adopting an informative FWCS (either multisensory or unimodal) that can benefit a broader range of driver demographics.

\section{ACKNOWLEDGEMENTS}

This research was supported by a grant (EP/J008001/1) from the EPSRC.

\section{REFERENCES}

Adams, A. S., \& Edworthy, J. (1995). Quantifying and predicting the effects of basic text display variables on the perceived urgency of warning labels: Tradeoffs involving font size, border weight and colour. Ergonomics, 38, 2221-2237.

Baldwin, C. L., \& Lewis, B. A. (2014). Perceived urgency mapping across modalities within a driving context. Applied Ergonomics, 45, 1270-1277.

Chun, J., Han, S. H., Pak, G., Seo, J., Lee I., \& Choi, S. (2012). Evaluation of vibrotactile feedback for forward collision warning on the steering wheel and seatbelt. International Journal of Industrial Ergonomics, 42, 443-448.

Chun, J., Lee, I., Park, G., Seo, J., Choi, S., \& Han, S. H. (2013). Efficacy of haptic blind spot warnings applied through a steering wheel or a seatbelt. Transportation Research Part F: Traffic Psychology and Behaviour, 21, 231-241.

Fagerlönn, J., Lindberg, S., \& Sirkka, A. (2012). Graded auditory warnings during in-vehicle use: Using sound to guide drivers without additional noise. In Proceedings of the 4th International Conference on Automotive User Interfaces and Interactive Vehicular Applications, 85-91.

Graham, R. (1999). Use of auditory icons as emergency warnings: Evaluation within a vehicle collision avoidance application. Ergonomics, 42, 1233-1248.

Gray, R. (2011). Looming auditory collision warnings for driving. Human Factors, 53, 63-74

Gray, R., Ho., C., \& Spence, C. (2014). A comparison of different informative vibrotactile forward collision warnings. PLoS ONE, 9: e87070.

Ho, C., Gray, R., \& Spence, C. (2014a). Reorienting driver attention with dynamic tactile cues. IEEE Transactions on Haptics, 7, 86-94.

Ho, C., Gray, R., \& Spence, C. (2014b). To what extent do the findings of laboratory-based spatial attention research apply to the real-world setting of driving? IEEE Transactions on Human-Machine Systems, 44, 524-530. 
Ho, C., Reed, N., \& Spence, C. (2007). Multisensory in-car warning signals for collision avoidance. Human Factors, 49, 1107-1114.

Ho, C., Santangelo, V., \& Spence, C. (2009). Multisensory warning signals: when spatial correspondence matters. Experimental Brain Research, 195, 261-272.

Ho, C., \& Spence, C. (2005). Assessing the effectiveness of various auditory cues in capturing a driver's visual attention. Journal of Experimental Psychology: Applied, 11, 157-174.

Ho, C., \& Spence, C. (2008). The multisensory driver: Implications for ergonomic car interface design. Aldershot: Ashgate Publishing.

Ho, C., Spence, C., \& Gray, R. (2013). Looming auditory and vibrotactile collision warnings for safe driving. Proceedings of 7th International Driving Symposium on Human Factors in Driver Assessment, Training, and Vehicle Design, 551-557, Bolton Landing, NY.

Kramer, A. F., Cassavaugh, N., Horrey, W. J., Becic, E., \& Mayhugh, J. L. (2007). Influence of age and proximity warnings devices on collision avoidance in simulated driving. Human Factors, 49, 935-949.

Lao, Y., Zhang, G., Wang, Y., \& Milton, J. (2014). Generalized nonlinear models for rear-end crash risk analysis. Accident Analysis \& Prevention, 62, 9-16.

Laughery, K. R. (2006). Safety communications: warnings. Applied Ergonomics, 37, 467-478.

Lee, J. D., McGehee, D. V., Brown, T. L., \& Reyes, M. L. (2002). Collision warning timing, driver distraction, and driver response to imminent rear-end collisions in a high-fidelity driving simulator. Human Factors, 44, 314-334.

Maltz, M., \& Shinar, D. (2007). Imperfect in-vehicle collision avoidance warning systems can aid distracted drivers. Transportation Research Part F: Traffic Psychology and Behaviour, 10, 345-357.

McGehee, D. V., Brown, T. L., Lee, J. D., \& Wilson, T. B. (2002). The effect of warning timing on collision avoidance behavior in a stationary lead vehicle scenario. In Human performance: Models intelligent vehicle initiative, traveller advisory and information systems (pp. 1-7). Washington, DC: Transportation Research Board.

Meng, F., Gray, R., Ho, C., Ahtamad, M., \& Spence, C. (2015a). Dynamic vibrotactile signals for forward collision warning systems. Human Factors, 57, 329-346.

Meng, F., Ho, C., Gray, R., \& Spence, C. (2015b). Dynamic vibrotactile warning signals for rear-end collision avoidance: Toward the torso vs. toward the head. Ergonomics, 58, 411 425 .

Mohebbi, R., Gray, R., \& Tan, H. Z. (2009). Driver reaction time to tactile and auditory rear-end collision warnings while talking on a cell phone. Human Factors, 51, 102-110

Murray, C. J., Vos, T., Lozano, R., Naghavi, M., Flaxman, A. D., Michaud, C., et al. (2012). Disability-adjusted life years (DALYs) for 291 diseases and injuries in 21 regions, 19902010: A systematic analysis for the Global Burden of Disease Study 2010. The Lancet, 380, 2197-2223.

Porter, M. M., Irani, P., \& Mondor, T. A. (2008). Effect of auditory road safety alerts on brake response times of younger and older male drivers: A simulator study. Transportation Research Record, 2069, 41-47.

Van Erp, J. B. F. (2005). Presenting directions with a vibrotactile torso display. Ergonomics, 48, 302-313.

Watanabe, Y., Ichikawa, H., Kayama, O., Ono, K., Kaneoka, K., \& Inami, S. (2000). Influence of seat characteristics on occupant motion in low-velocity rear-end impacts. Accident Analysis \& Prevention, 32, 243-250. 